\title{
Reduction of a Cabin Thermal Bond Graph Model
}

\author{
Youssef Hammadi*, David Ryckelynck** and Amin EL-BAKKALI*** \\ * Methods and Tools for Numerical Simulation Department, Renault Group, Guyancourt, France, \\ (e-mail: youssef.hammadi@renault.com,youssef.hammadi@mines-paristech.fr). \\ ** Simulations of Materials and Structures Department, MINES ParisTech Center of Material, Evry, France, \\ (e-mail: david.ryckelynck@mines-paristech.fr). \\ *** Methods and Tools for Numerical Simulation Department, Renault Group, Guyancourt, France, \\ (e-mail: amin.el-bakkali@renault.com).
}

Keywords: model reduction, automotive control, machine learning, singular value decomposition, bond graphs, differential equations

\section{INTRODUCTION}

In order to describe the transient thermal behavior of an automotive cabin, we need to model the energy and mass exchanges between the cabin system and the environment as well as these exchanges inside the cabin system itself.

Given that the cabin model and its environment are intended to be integrated into a control loop, we have adopted a system level modelling (0D) based on the Bond Graph approach [Paytner 1961]. The simulator, thus built, results in a set of nonlinear Differential Algebraic Equations (DAE). As part of this work, we will use the Discrete Empirical Interpolation Method (DEIM) [Chaturantabut 2010] to solve DAE only for a selection of explanatory variables. This is a way to build a Reduced Bond Graph by decreasing the number of solved differential equations.

\section{CABIN THERMAL MODEL AND REDUCTION METHODOLOGY}

\subsection{Cabin thermal model}

In bond graph approach applied to thermal domain, two elements exchanging an energy are linked by a line (Bond). The heat flux exchanged between the two elements is expressed as the product of the temperature $\theta$ (effort variable) and the entropy flux $\dot{s}$ (flow variable). The line is also completed with a half-headed arrow indicating the positive direction of heat transfer, and a causal line indicating which of the two elements receives the effort variable and returns the flow one.

Figure 1 shows the bong graph associated to the thermal conduction through a wall represented by an internal and an external thermal capacitance connected through an equivalent thermal conductance. $\theta_{w i}$ and $\theta_{w e}$ are respectively the temperatures of the internal and external surfaces of the wall. $\dot{s}_{\text {int }}$ and $\dot{s}_{\text {ext }}$ are respectively the internal and external entropy fluxes.

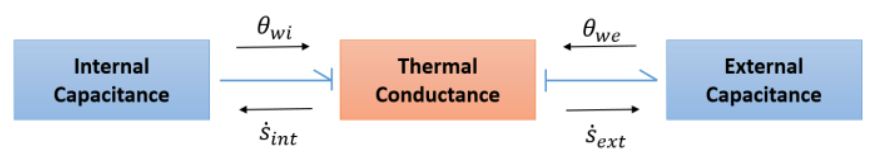

Fig. 1. Bond graph modelling of thermal conduction through a wall.

For a cabin system containing a number $N w$ of walls and a number $N a$ of air zones, the global thermal bond graph leads to the nonlinear first order DAE system (1):

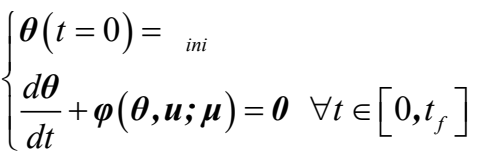

$$
\begin{aligned}
& \boldsymbol{\theta}=\left[\begin{array} { l } 
{ [ \boldsymbol { \theta } _ { \boldsymbol { w } } ] } \\
{ [ \boldsymbol { \theta } _ { \boldsymbol { a } } ] }
\end{array} \quad \mathbb { R } ^ { N } \text { with } \left\{\begin{array}{ccc}
\boldsymbol{\theta}_{\boldsymbol{w}}=\left[\begin{array}{c}
\left\lceil\theta_{w i_{1}}\right. \\
\left\lfloor\theta_{w_{1}}\right.
\end{array}\right] & \left.\cdots\left[\begin{array}{c}
\theta_{w i_{N w}} \\
\theta_{w e_{N w}}
\end{array}\right]\right]^{T} \\
\boldsymbol{\theta}_{\boldsymbol{a}}=\left[\begin{array}{c}
{\left[\theta_{a_{1}}\right.} \\
x_{1}
\end{array}\right] & \left.\cdots\left[\begin{array}{c}
\theta_{a_{N a}} \\
x_{N a}
\end{array}\right]\right]^{\boldsymbol{T}}
\end{array}\right.\right.
\end{aligned}
$$

Where $\quad N=2(N w+N a), \quad \boldsymbol{\theta} \in \mathbb{R}^{N} \quad$ is the unknown temperatures and absolute humidities vector defined by (2), $\boldsymbol{\varphi}(\boldsymbol{\theta}, \boldsymbol{u} ; \boldsymbol{\mu}) \in \mathbb{R}^{N}$ is a nonlinear function deduced from energy and mass balances, $\boldsymbol{u} \in \mathbb{R}^{N u}$ contains the bong graph input variables, $\boldsymbol{\mu} \in \mathbb{R}^{N \mu}$ is a set of the bond graph parameters, $\boldsymbol{\theta}_{w} \in \mathbb{R}^{2 N w}$ is the vector of all walls internal $\theta_{w i}$ and external $\theta_{\text {we }}$ temperatures, and $\boldsymbol{\theta}_{\boldsymbol{a}} \in \mathbb{R}^{2 N a}$ is the vector of all air zones temperatures $\theta_{a}$ and absolute humidities $x$.

\subsection{Reduction Approach}

In order to build a Reduced Order Model (ROM), we need to construct a matrix of all possible responses of the studied system, and then extract some empirical modes from this matrix. However, such a matrix requires an infinite memory 
storage size if all possible parametric and input predictions are considered.

The model reduction approach, proposed here, begins with an unsupervised machine learning phase to develop the Reduced Order Model (ROM), followed by an online phase to use the reduced model already built. During the machine learning phase, a reduced basis $\boldsymbol{V} \in \mathbb{R}^{\mathrm{N} \times \mathrm{n}}$ is constructed using an $n$ order Truncated Singular Value Decomposition (Truncated SVD), also called Principal Component Analysis (PCA), applied to a matrix of $L$ different input and parameter sets simulations, randomly selected. This matrix $\boldsymbol{A} \in \mathbb{R}^{\mathrm{N} \times(L m)}$, where $m$ is the time points number for each simulation, is defined by:

$$
\begin{aligned}
& A_{i j}=\theta_{i}^{(l)}\left(t_{k}\right)-\theta_{i n i}^{(l)} \\
& \text { with } j=(l-l) m+k, \quad 1 \leq k \leq m, \quad 1 \leq l \leq L .
\end{aligned}
$$

Where $\theta_{i}^{(l)}\left(t_{k}\right)$ represents the $i^{t h}$ element of the unknown vector at time $t_{k}$ using the system inputs and parameters $\left(u^{(l)} ; \mu^{(l)}\right)$ which are generated from a Design Of Experiment (DOE). We point out that subtracting the initial temperature in (3) is a trick that we propose to make the initial error null.

The reduced basis construction is then completed by a selection of state variables: using the DEIM, we obtain a list of interpolation indexes that we complete with some air zones indexes to form thereby the list $\mathcal{P}$. Our reduction approach consists of choosing the temperatures $\boldsymbol{\theta}[\wp]$ as the ROM explanatory variables. We write $\boldsymbol{\theta}_{r}$ these variables, which satisfy the following equations:

$$
\begin{aligned}
& \boldsymbol{\theta}=\hat{\boldsymbol{V}} \theta_{r} \\
& \hat{\boldsymbol{V}}[\wp,:]=\boldsymbol{I} \\
& \hat{\boldsymbol{V}}\left[\Re,:\left[=\boldsymbol{V}[\Re,:]\left(( \boldsymbol { V } [ \wp , : ] ) ^ { T } \boldsymbol { V } \left[\wp,:[)^{-1}(\boldsymbol{V}[\wp,:])^{T}\right.\right.\right.\right. \\
& \mathfrak{R}=\{1, \ldots, N\} \mid \wp
\end{aligned}
$$

Where (6) is given by the Gappy POD method [Everson 1995] which allows to reconstruct a field by measuring it only in some explanatory points that form the Reduced Integration Domain (RID) [Ryckelynck 2005].

In practice, this approach is non-intrusive for bond graphs since we just need to add the linear constraint $\boldsymbol{\theta}[\mathfrak{R}](t)=\hat{\boldsymbol{V}}[\mathfrak{R},:] \boldsymbol{\theta}[\wp](t)$.

The ROM DAE system (8) is then obtained by projecting the original DAE system (1) onto the subspace $\hat{\boldsymbol{V}}[\wp,:]=\boldsymbol{I}$ :

$$
\left\{\begin{array}{l}
\boldsymbol{\theta}_{r}(t=0)=\boldsymbol{\theta}_{i n i}[\wp] \\
\frac{d}{d t}\left(\hat{\boldsymbol{V}}[\wp,:] \boldsymbol{\theta}_{r}\right)+\boldsymbol{\varphi}[\wp]\left(\hat{\boldsymbol{V}} \boldsymbol{\theta}_{r}, \boldsymbol{u} ; \boldsymbol{\mu}\right)=\boldsymbol{0} \quad \forall t \in\left[0, t_{f}\right]
\end{array}\right.
$$

If we want to build a Reduced Bond Graph at this stage, we will need to reconstruct all walls internal temperatures, which limits the ROM speedup. In fact, these temperatures are necessary to calculate the total internal convective heat flux received by walls-adjacent air zones, and then determine their temperatures. In a perspective similar to [Ryckelynck 2015] where displacements as well as stresses fields are used to build a hyper-reduced model, we propose to perform, in addition to temperature variables reduction based on the matrix $\boldsymbol{A}$, a similar treatment on internal convective heat flux variables in order to reduce the computational time related to internal convective heat fluxes calculation.

\section{APPLICATION}

We apply here the reduction approach to a cabin thermal model with $N w=18, N a=1, n=6, t_{f}=14400 \mathrm{~s}$, and by varying 8 continuous inputs and parameters: vehicle speed, ambient temperature, vehicle initial temperature, ambient humidity, solar irradiance as well as the mass flow rate, humidity and temperature of the air supplied by AC system. We note that, for this application, only internal and external walls temperatures were exported to form the matrix $\boldsymbol{A}$.

We obtain a CPU time reduction by $30 \%$. Concerning the accuracy of the ROM, we generate a new DOE of 500 points, and then draw a histogram of simulations mean squared errors on air zone temperature, which is shown in Figure 2.

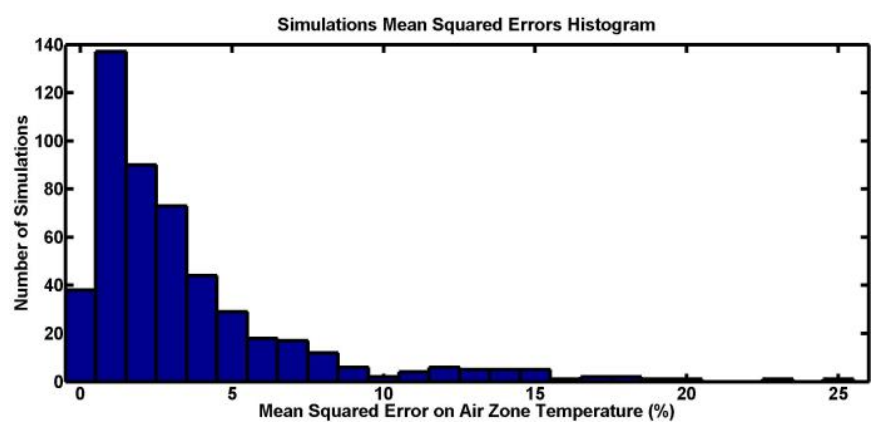

Fig. 2. Simulations mean squared errors histogram

Among the 500 simulations launched, 477 simulations are characterized by a mean squared error of less than $12.5 \%$ (equivalent to $1 \mathrm{~K}$ ), which is sufficient for air zone temperature regulation scenarios.

\section{REFERENCES}

Chaturantabut, S. and Sorensen, D.C. (2010). Nonlinear model reduction via discrete empirical interpolation. SIAM J. Sci. Comput., 32(5), 2737-2764.

Everson, R. and Sirovich, L. (1995). Karhunen-Loève procedure for gappy data. Journal of the optical society of America A, 12(8), 1657-1664.

Paytner, H.M. (1961). Analysis and design of engineering systems. The MIT press.

Ryckelynck, D. (2005). A priori hypereduction method: an adaptive approach. International Journal of Computational Physics, 202, 346-366.

Ryckelynck, D., Lampoh, K., and Quilici, S. (2015). Hyperreduced predictions for lifetime assessment of elastoplastic structures. Meccanica, 51(2), 309-317. 